\title{
Hepatocellular carcinoma care during The Coronavirus disease (COVID-19) era
}

\author{
Ali Abdel Aziz Ali*, Hammam Abo elalamin Elsaid, Ghada \\ Moustafa kamal Galal, Khairy Hammam Morsy. \\ Department of Tropical Medicine and Gastroenterology, Faculty of \\ Medicine, Sohag University
}

\begin{abstract}
:
The coronavirus pandemic (COVID-19) is now a great universal health problem and the biggest threat we have to compete since World War Two. Since its emergence in China late last year, this virus has reached to all world continents except Antarctica. Cases in Europe, the Americas, and Africa are growing regularly. COVID-19 pandemic is spreading like a wave-one that may yet crash on those least able to face, accounting a much more than just a health issue. All medical communities are facing a lot of challenges either in preventing or treating this infection.COVID-19 is a hazardous infection especially in cancer patients that need a more immediate priority in obtaining medical care. Liver cancer is the world's sixth most common malignant tumor and the fourth common malignant tumor in Egypt, also, Egypt is the third and fifteenth most crowded region in Africa and globally. This review seeks to highlight the best measures that cut the risk of deterioration in liver cancer patients leading to the best outcome in facing the COVID-19 pandemic.

Keywords: Keywords: coronavirus (COVID-19) pandemic, Hepatocellular carcinoma (HCC), novel coronavirus.

Abbreviations: Abbreviations: ALF: acute liver failure, COVID-19: Coronavirus disease, HCC: Hepatocellular carcinoma, MERS-CoV: Middle East respiratory syndrome. RNA: ribonucleic acid, PPE: Personal protective equipment, SARS-CoV(2): severe acute respiratory syndrome coronavirus type 2 .
\end{abstract}

\section{Introduction:}

Coronavirus disease-19 (COVID-19) is an infectious disease transmitted by the rapidly spreading novel coronavirus overall the globe (1). All doctors must do their best to run in their diseased people, practice offices, and health institutes for the crucial impacts that may overwhelm the capability of our healthcare system and for proposed measures that we need to limit disseminate of SARS-CoV(2) (2). On the other hand, They must persist to pay the care for the patients suffering from chronic liver disease, liver cancer, and hepatic transplant, where special logistical and pharmacological challenges occur, so All hepatologists seek to have a clear framework for developing practical guidance and concerns reducing the effects of the COVID-19 dissemination on people with liver disease and health care professionals (3).

\section{COVID-19}

The novel coronavirus that causes COVID-19 may cause death in 2-3\% of infected people, with the increased possibility of fatal complications in the old age and patients with chronic illness (4).

Coronaviruses have seven identified Species that infect human beings; SARS-CoV, MERS-CoV, and n-CoV can cause serious disease, while others 
have mild symptoms (5). $\mathrm{n}-\mathrm{CoV}$ is an RNA-virus and also called SARSCoV-2(6).

In humans, $\mathrm{n}-\mathrm{CoV}$ is very contagious, and spreading from person to others is effective and reported by WHO that document multiple outbreaks in several areas. On lung imaging studies, COVID-19 is characterized by multiple areas of ground-glass opacities, that also may occur in patients with mild picture (7).

As of April 8, 2020, SARS-CoV-2 transmitted to more than $1,300,000$ persons, and 72,500 have died worldwide since the earliest record of new respiratory illness in the Wuhan region, China. (8).

\section{SARS-CoV-2 pathogenesis}

The exact mechanism of infection with $\mathrm{n}-\mathrm{CoV}$ in man stills unknown. The immunity role is the powerful protection opposite to any invading pathogenic agent and no effective antiviral therapy act specifically against the virus at present(9).

The immune system does a critical position in protecting from $\mathrm{n}-\mathrm{CoV}$ and other coronaviruses infections. Immunity sequential effects in SARS, MERS, and influenza patients, including alterations in $\mathrm{T}$ lymphocyte clades peripherally that help to understand the special properties of the disease, identification, assessment, prevention, and efficient therapy (10).

The clinical course having three stages: viremia, acute (pneumonia), and recovery stage. If patients' immune function in the 1st stage is successful, Furthermore without any significant illness, the immunity can efficiently block the Viral load and clears the virus, then he will be cured. If the person is elderly, immunecompromised, or has another comorbidity like diabetes, renal failure, the immunity couldn't efficiently stop the virus during the 1st stage (pneumonia stage), the patient's condition may become serious or crucial. According to this immune theory, $\mathrm{T}$ cells, B cells were moreover decreased, where the mediators of inflammation, cytokines, and D-Dimer elevate continuously during the severe course of the disease(9).

\section{Immunity changes in hepatocellular carcinoma}

The immune system has a strong impact on the pathogenesis of many tumors. Compelling evidence shows that cancer evades destruction by suppressing the host's immune system. Currently, most pathologists accepted that interactions between tumorimmune cells are generally highly important for survival (11).

For cases with a more serious picture of the disease, depression of the immune system can also offer a little defense from immunopathogenesis, which tends to result in more pulmonary insult. This can be related to a macrophages stimulation syndrome in a hyper inflammatory state Distinguished by a multi-organ cytokines storm leading to more multiple organ damage (12).

\section{Outpatient care maintainance}

The capacity of the outpatient clinic should be limited to allow a person to keep a gap of at least $1 \mathrm{mt}$ between the patients in the waiting room, Patients with chronic and worsening liver disease are often treated and tracked in bigger unities or centers. Nevertheless, these hospitals are reportedly also coronavirus risky areas, placing outpatients with hepatic disorders at great hazard for nosocomial infections(13).

Additionally, Prioritization issues with outpatient interactions will certainly be discussed. The exact care of these patients varies according to the regional COVID-19 dissemination (13). 


\section{Measures when suspect infection in $\mathrm{HCC}$ patient}

- Search for signs of COVID-19 or earlier contact (i.e. cough, high temperature, interaction with established COVID-19 patients, returned traveler from an outbreak area) before accessing clinical units (i.e. phone call 24 hours before scheduled visits), and once more at strict recording or when they reach the institution(14).

- Measure temperature for every patient upon Emergence at the clinic or check-in desk. - Refer to hightemperature patients $\left(>100^{\circ} \mathrm{F}\right)$ to the triag field and treated for symptomatic patients according to local guidelines.

- Evaluate all symptomatic infected persons with COVID-19 at an ambulatory clinic or designated area for this reason. provide this location with all personal protective equipment (PPE). Never test a suspected infected person in any clinic for hepatoma or liver transplants whether symptomatic or not (13).

- Maintain physical distancing to lower the transmission of $\mathrm{n}-\mathrm{CoV}$ infection. This successful approach suggested preventing a fast rise in the general population of SARS-CoV-2 infections with the final target of reducing the bulk of patients with a serious COVID19 picture for all times, helping health care providers to improve their medical/intensive care. Parallel to this, doctors should cover the susceptible patient against persons with possible exposure or infection with SARSCoV-2. So they must compare the advantages of improving patient treatment and the possible risk of infection in such patients (14).

These requirements include the power to introduce standardized operating rules for outpatient treatment, includes the remodeling of waiting sectors to enable enough room, restricting waiting periods, and enabling patients to wait for externally when necessary and to be called by telephone at last. Moreover, restrict medical staff exposure where possible, direct interaction is the basis for the relationships between doctor and patient and helps doctors to easily explain the patient's overall condition (15).

Encourage medical authorities to equip hospitals and clinics with these measures not just to care for COVID19 patients who still quarantined at home, but also to care for persons who need doctors help for a strongly dangerous infection and to guide them through the infection time, describing possible management strategies and plans and Provide guidance on systematic preventive measures(13).

\section{HCC screening}

Doctors better postpone HCC screening at the unit and personal risk evaluation depending on service availability and Higher-risk conditions such as advanced cirrhosis, higher levels of alpha-fetoprotein, NASH/diabetes, etc. (14).

\section{Suspected/confirmed HCC}

Patients non-COVID-19:

- Review of all radiological data referred recently for patients with liver focal lesions in hepatoma unit or with professional radiologists for an inperson appointment schedule. Allow virtual meetings to discuss treatment, and discuss HCC and other liver tumor possibilities.

Exactly, these diseased persons will die before the pandemic eradicates, since no one can expect the prospective pandemic timeframe. Arbitrary delay of 2 months is fair, based on the patient and facility-linked situation. - Establish therapies for liver cancer, rather than postpone them explanatory by the COVID-19 pandemic (16).

Patients having COVID-19: 
- Postpone locoregional treatments wherever possible, and gradually remove anti-cancer immunological treatment. In non-severe COVID-19, the decision on whether to continue sorafenib treatment (low dosage) should doctors take on a situation-bycase basis (16).

\section{HCC patient with hepatic decompensation:}

- Maintain medical services according to established protocol, but it is important to allow minimal access to doctors by the use of telemedicine/phone visits wherever available/needed to evade direct contact(13).

- Limit the listing of transplants to people with poor outcome and survival such as patients with acute liver failure (ALF), severe acute exacerbation of chronic liver disease, high end-stage liver disease model (MELD) scores (includes exceptional MELDs) and HCC at the upper limits of the Milan criterion, as transplantation effortsliver donations are likely to be decreased in several regions(16).

- Encourage pneumococcal and influenza vaccinations. - Strict apply the issues relating to general proph-ylaxis of spontaneous bacterial peritonitis and hepatic coma to limit deterioration and evade admission requests. - Perform SARS-CoV(2) checking to all patients with acute decompensation, exacerbation, or acute on top of chronic liver failure (16).

\section{Upper GIT endoscopy for HCC patients:}

Non-urgently needed: Doctors should restrict endoscopic manipulation when possible according to Baveno consensus(13).

Urgently needed: Doctors must take all personal and environmental protective precautions to decrease the possibilities of COVID-19 transm-ission within the endoscopy department(17).

\section{HCC patient listed for transplantation:}

- Apply the routine testing of COVID19 in both recipients and donors before transplantation, understanding that negative results cann't prevent the disease entirely. Consent measures will guarantee the possible risk of nosocomial COVID-19 for anyways for diagnosis or treatment relevant to transplantation. ${ }^{-} \quad$ Living-donor transplants best-individualized Build on a state by state basis (3).

\section{Post liver transplantation immunosuppression:}

Chinese doctors recommended using corticosteroids in briefly in treatment of COVID-19 in contrast, WHO not recommend that while stopping immunosuppression may lead to many complications EASL guidelines recommend the limitation of immunosuppressive therapy (18).

\section{Inpatient care maintenance}

During the coronavirus pandemic a significant number of patients with a chronic hepatic illness will continue to need inpatient treatment for decomppensation, acute insult, rejection, or Additional health problems. General concerns for these risky patients to protect them from exposure to SARSCoV-2 as acquired COVID-19 may be of critical significance(3).

Medical care professionals need to adopt COVID-19-clean wards or hospitals based on the local infras-tructure. However, doctors have difficulty to reach a definite distinction of clean and dirty wards/hospitals depending on the local COVID-19-burden, although new research gives somewhat promising data that SARS-CoV-2 RNA is not found on inanimate surfaces outside the patient's room. Wherever practical, they should define the inpatient treatment requirements for non-COVID19 reasons as COVID-19-clean wards or hospitals for patients with HCC and/or 
chronic liver disease. Since these areas may fail to offer comprehensive hepatology care, we suggest that Expert institutions have readily available contact information to ease prompt consultations on liver diseases (19).

\section{Presumed treatments of COVID-19 in HCC patient}

- Ensure early admission to lowers impacts of potential risk factors and use of COVID-19 in clinical trials and experimental anti-viral treatment as per local protocols. Warning regard for excessive doses of paracetamol (2-3 $\mathrm{gm} /$ day is usually considered not harmful in non-alcoholic patients with cirrhosis)(16).

- For patients with liver cirrhosis and portal hypertension, stop using salicylates and other NSAID medications(20).

- Novel clinical trials will not start now unless they meet the concept of an appropriate clinical trial that matches with regional issues. Consider deferral of any other clinical study visits in person(16).

- According to numerous clinical trials lopinavir/ritonavir fails to give a more beneficial effect in severe infection with multiple side effects and better avoid(21).

\section{Conclusion}

HCC patients considered a fragile population of patients with a higher possibility of catching the infection and/or extreme COVID-19 progression. Moreover, the growing disseminated situation worldwide needs an unprecedented allocation of health-care services that can adversely impact the medical treatment of chronic hepatic and cancer liver patients who continue to seek medical care.

\section{References}

1- Guan W, Ni Z, Hu Y, Liang WH, Ou CQ, He JX, et al. Clinical characteristics of coronavirus disease 2019 in China. N Engl J Med 2020 Feb 28. doi: 10.1056/NEJMoa2002032.

2- Chopra V, Toner E, Waldhorn R, Washer L. How should U.S. hospitals prepare for coronavirus disease 2019 (COVID-19)? Ann Intern Med 2020 Mar 11. doi: 10.7326/M20-0907.

3- Stephen Chan, Joong-Won Park, MANAGEMENT OF HCC DURING COVID-19: ILCA GUIDANCE, 8th April 2020.

4- Wu Z, McGoogan JM Characteristics of and important lessons from the coronavirus disease 2019 (COVID-19) outbreak in China: summary of a report of 72314 cases from the Chinese Center for Disease Control and Prevention. JAMA. 2020; (published online Feb DOI:10.1001/jama.2020.2648

5- Corman, V. M., Muth, D., Niemeyer, D. \& Drosten, C. Adv. Virus Res. 100, 163-188 (2018).

6- Chen N, Zhou M, Dong X, Qu J, Gong F, Han Y, et al. (15 February 2020). "Epidemiological and clinical characteristics of 99 cases of 2019 novel coronavirus pneumonia in Wuhan, China: a descriptive study". The Lancet. 395 (10223): 507513. doi:10.1016/S0140-

6736(20)30211-

7. PMID 32007143. Archived from the original on 31 January 2020. Retrieved 9 March 2020.

7- Chan JF-W, Yuan S, Kok K-H, et al.A familial cluster of pneumonia associated with the 2019 novel coronavirus indicating person-toperson transmission: a study of a family cluster.Lancet. 2020; 395: 514523.

8- World Health Organization, 8 April 2020.

9- Taisheng $L$, Zhifeng Q, Linqi Z, et al. Significant changes of peripheral T lymphocyte subsets in patients with severe acute respiratory syndrome. J Infect Dis. 2004;189(4):648-651. doi: 10.1086/381535.

10- Ling Lin, Lianfeng Lu, Wei Cao \& Taisheng Li Emerg Microbes Infect. 2020 Dec;9(1):727-732. doi: $10.1080 / 22221751.2020 .1746199$. 
11- Bindea G, et al. Spatiotemporal dynamics of intratumoral immune cells reveal the immune landscape in human cancer. Immunity. 2013;39:782-795. doi: 10.1016/j.immuni.2013.10.003.

12- Li X, Geng M, Peng Y, Meng L, Lu S. Molecular immune pathogenesis and diagnosis of COVID19. Journal of Pharmaceutical Analysis 2020.

13- Boettler T, Newsome PN, Mondelli MU, Maticic M, Cordero E, Cornberg M, Berg T, Care of patients with liver disease during the COVID19 pandemic: EASL-ESCMID position paper, JHEP Reports (2020)

14- Wong, S.H., R.N. Lui, and J.J. Sung, Covid-19 and the Digestive System. J Gastroenterol Hepatol, 2020.

15- Hollander JE, Carr BG. Virtually Perfect? Telemedicine for Covid-19. New England Journal of Medicine 2020.

16- American Association for the Study of Liver Diseases WWW.AASLD.org | Released March 23, 2020.
17- Repici A, Maselli r, Colombo M, Gabbiadini R, et al., Coronavirus (COVID-19) outbreak: what the department of endoscopy should know. Gastrointestinal endoscopy 2020.

18- Hongling Liu, $\mathrm{Xi} \mathrm{He}$, et al., Management of COVID-19 in patients after liver transplantation: Hepatol Int. 10Apr 2020: 1-5.

19- Colaneri M, Seminari E, Piralla A, Zuccaro V, Filippo AD, Baldanti F, et al. Lack of SARS-CoV-2 RNA environmental contamination in a tertiary referral hospital for infectious diseases in Northern Italy. Journal of Hospital Infection.

20- Chandok N, Watt KDS. Pain Management in the Cirrhotic Patient: The Clinical Challenge. Mayo Clinic Proceedings 2010;85:451-458.

21- Cao B, Wang Y, Wen D, et al. A trial of lopinavir-ritonavir in adults hospitalized with severe Covid-19. N Engl J Med. 2020 doi: 10.1056/NEJMoa2001282. 